\title{
Les effets au niveau national et régional de la coopération internationale des polices : un système spécifique de multi-level governance
}

\section{Hartmut Aden}

\section{(2) OpenEdition \\ Journals}

Édition électronique

URL : http://journals.openedition.org/conflits/899

DOI : $10.4000 /$ conflits.899

ISSN : 1777-5345

Éditeur :

CCLS - Centre d'études sur les conflits lilberté et sécurité, L'Harmattan

Édition imprimée

Date de publication : 1 décembre 2002

ISBN : 2-7475-4291-2

ISSN : $1157-996 \mathrm{X}$

\section{Référence électronique}

Hartmut Aden, "Les effets au niveau national et régional de la coopération internationale des polices un système spécifique de multi-level governance », Cultures \& Conflits [En ligne], 48 | hiver 2002, mis en ligne le 27 avril 2003, consulté le 30 mars 2021. URL : http://journals.openedition.org/conflits/899 DOI : https://doi.org/10.4000/conflits.899

Ce document a été généré automatiquement le 30 mars 2021.

Creative Commons License 


\title{
Les effets au niveau national et régional de la coopération internationale des polices : un système spécifique de multi-level governance
}

\author{
Hartmut Aden
}

1 Europol, Interpol et la plupart des autres structures de la coopération internationale des polices sont relativement éloignées du travail policier au quotidien ${ }^{1}$ Néanmoins, ces formes de coopération ont des impacts dont la portée dépend des structures et des caractéristiques des systèmes de police préexistants au niveau national, régional et local. Dans une perspective théorique, les différents niveaux administratifs et politiques concernés par les aspects internationaux du travail policier peuvent être analysés comme un système de multi-level governance. Ce concept a été développé pour étudier les formes spécifiques de coordination et de conflits entre les acteurs politiques, bureaucratiques et privés dans des systèmes «à plusieurs niveaux" géographiques dont le prototype est la Communauté Européenne voire l'Union Européenne $^{2}$. Il a été relativement peu appliqué au cas de la coopération policière jusqu'ici. Il s'y apprête parce que les différents niveaux administratifs et politiques y sont interconnectés dans les deux directions, mais dans des logiques asymétriques. Cela est le cas non pas seulement pour la préparation des initiatives politiques, mais surtout pour leur mise en oeuvre.

2 L'analyse suivante va d'abord présenter les raisons de la distance importante des institutions de la coopération internationale du travail policier au quotidien. Ensuite, elle va analyser les différents types d'impacts que la coopération internationale a au niveau national : les effets centralisateurs, l'élargissement du domaine autonome de la police par rapport à la justice, les effets de synergie et de modernisation au niveau de la police technique, le gain d'importance de certaines formes d'investigation 
«undercover », les impacts au niveau du droit et de la criminalité et finalement les impacts sur les professions policières et sur un contrôle démocratique des polices.

Sphère autonome ou élément du travail policier quotidien ? Les limites des impacts au niveau national de la coopération internationale

3 La coopération internationale des polices n'implique directement qu'une petite partie des policiers dans les pays qui y participent. Ce sont surtout les élites de la police criminelle qui dominent les négociations officielles et la mise en oeuvre de la coopération. Interpol, créé en 1923, a toujours été une sorte de "policemen's club» fondé à l'initiative de dirigeants policiers. Les gouvernements n'y ont joué qu'un rôle secondaire. La coopération internationale a donc tendance à être l'affaire d'un petit cercle d'experts, souvent motivés par des sentiments d'avant-garde pour la lutte contre le crime international. Avec la création d'Europol, cette tendance s'est renforcée en Europe. Europol est basé sur un concept élitiste d'analyse du renseignement. Le processus d'analyse des données est strictement secret ${ }^{3}$, non pas seulement pour des raisons de protection des données, mais surtout à cause de la crainte de fuites d'informations vers le milieu criminel. Sous cet aspect-là, la coopération internationale des polices est en effet une sphère relativement autonome. La conception d'Europol tente d'adoucir cet éloignement en créant une autre banque de données avec des informations moins sensibles de recherche et de référence consultable par les unités centrales de police criminelle des pays membres ${ }^{4}$. Néanmoins Europol fait partie d'une bureaucratie d'investigation criminelle autoréférentielle et relativement fermée dans ses opérations. Ce système ${ }^{5}$ fonctionne sur la base de ses propres cercles de communication et il ne renoue les liens vers l'extérieur que pour recueillir du renseignement, pour chercher la coopération avec les unités de police criminelle dans les pays membres et pour propager les succès de son travail ou pour demander plus de soutien de la part des responsables politiques.

4 En règle générale le policier « de base » qui patrouille dans une ville quelque part en Europe ne connaît les institutions de la coopération internationale que de loin. Les idées qu'il s'en fait proviennent principalement des récits le plus souvent peu systématiques qu'il trouve dans les revues des syndicats ou dans les médias de grande diffusion. Mais lors d'un contrôle d'identité ou d'une vérification de la plaque d'immatriculation d'une voiture, les impacts de cette coopération sont évidents. Depuis le milieu des années 1990 les policiers se connectent à une banque de données élargie à des personnes voire des voitures recherchées dans d'autres pays européens dans le cadre des accords de Schengen. Même si la provenance de ces données n'est en principe pas importante pour le travail policier au quotidien, celui-ci doit a la coopération internationale un élargissement considérable du renseignement dont il peut se servir.

5 Dans les régions frontalières, le cas est différent. Près des frontières, les policiers se voient régulièrement confrontés à la question de la conduite à tenir dans un cas qui se révèle avoir des liens avec le pays voisin. La poursuite de l'auteur d'un hold-up qui fuit au-delà de la frontière en est l'exemple le plus parlant et donc le plus souvent cité. A cet égard, dans la région frontalière entre les Pays-Bas, la Belgique et l'Allemagne autour de Maastricht et Aix-la-Chapelle, des dirigeants policiers ont créé en 1979 déjà NEBEDEAG-POL, une structure de governance autogérée sur la base d'une association de droit privé, pour mieux coordonner leur travail ${ }^{6}$. Avec les accords de Schengen, ce genre de coopération transfrontalière est devenue une stratégie officielle. Elle a été institutionnalisée dans un nombre considérable de centres policiers et douaniers le 
long des frontières dans lesquelles coopèrent les fonctionnaires des pays voisins, par exemple entre la France et l'Allemagne avec le Centre de Coopération Policière et Douanière à Offenburg qui travaille sur la base d'un accord entre les deux pays en date du 9 octobre 1997.

Si la coopération internationale des polices est une sphère relativement autonome, elle ne pourrait pourtant pas exister sans un minimum d'utilité pratique pour le travail policier dans les pays membres. Dans une perspective de longue durée il ne suffirait pas de légitimer l'existence d'une institution de coopération uniquement par sa fonction de structure bureaucratique "en réserve" à laquelle les acteurs politiques peuvent déléguer la responsabilité pour tout genre de problème de sécurité qui pourrait surgir ${ }^{7}$. Ces institutions doivent donc se légitimer surtout par leur utilité pour les investigations dans des cas concrets, non pas seulement dans les régions frontalières. $\mathrm{Si}$ elles n'y arrivent pas, elles auront au minimum des difficultés à débloquer des crédits pour de nouvelles activités.

L'organisation policière : les effets centralisateurs

$7 \quad$ Il y a plusieurs facteurs qui peuvent contribuer à mesurer le degré de centralisation ou de décentralisation d'une administration : le degré de dépendance ou d'autonomie dont une administration décentralisée dispose par rapport aux administrations centrales, la distance entre l'administration et les problèmes à résoudre, l'importance des structures centralisées par rapport aux structures décentralisées, la répartition des ressources financières, etc. En pratique, la centralisation ou décentralisation d'une administration varie selon la taille d'un pays et d'autres facteurs comme par exemple le rôle de l'État dans la structuration de la vie quotidienne. L'effet centralisateur que la coopération internationale des polices peut avoir dans un petit pays avec une administration traditionnellement décentralisée est donc plus important que dans un pays dont les administrations sont déjà centralisées depuis longtemps ${ }^{8}$. Un processus de centralisation n'est en général pas linéaire, mais à longue échéance, la centralisation provoque souvent des résistances et par la suite des tendances inverses vers une décentralisation ${ }^{9}$.

8 Pour les structures de police, Fijnaut a distingué cinq facteurs qui ont contribué à leur centralisation depuis le milieu du 19ème siècle : Le renforcement des unités de police le plus souvent paramilitaires des Etats centralisés, la création de services spécialisés, l'étatisation de structures policières locales, l'augmentation de l'influence de l'État central par un cofinancement des structures décentralisées et la disparition de structures de police communales ${ }^{10}$. Si les polices ont eu une tendance prédominante à la centralisation, cela est surtout dû à deux types de causes : Premièrement, l'intérêt des élites politiques au niveau des Etats centraux à disposer d'une force de police paramilitaire. Cet intérêt a souvent mené à une centralisation massive des structures de police ou à la création de nouvelles structures centralisées. Deuxièmement, le fait que les structures centralisées une fois installées ont souvent tendance à élargir leurs missions et leurs effectifs dans une logique de dynamique autonome. Les fonctionnaires voient d'autres problèmes qu'ils pourraient résoudre. L'élargissement des structures administratives améliore les perspectives de carrière. Cette dynamique autonome coïncide bien avec l'intérêt des responsables politiques à disposer d'une institution à laquelle ils peuvent déléguer tout problème de sécurité qui peut surgir ${ }^{11}$.

9 Au niveau de l'organisation policière, la coopération internationale a augmenté le poids des services centralisés. Cette tendance n'est pas nouvelle. La création d'un Bureau 
central national $(\mathrm{BCN})$ était déjà une condition préalable pour la participation aux activités d'Interpol. Dans des pays comme la République Fédérale d'Allemagne ou les Pays-Bas dont les structures policières étaient pendant longtemps relativement décentralisées, la coopération avec Interpol fut pendant longtemps la mission principale des services centralisés de la police criminelle. Les formes de coopération des polices en Europe créées depuis les années 1970 ont accéléré ce processus. Pour participer au groupe Schengen, chaque pays signataire a dû créer une banque de données centralisée pour se connecter au Système Informatique Schengen (SIS) et une unité SIRENE ${ }^{12}$ qui gère le flux de renseignement entre les unités de base et Schengen. Europol demande également dans chaque pays un bureau unique qui assure la coopération avec toutes les unités de police de ce pays ${ }^{13}$. Le plus souvent, toutes ces structures ont été concentrées dans un seul service qui a gagné par cette voie un poids important. C'est par exemple le cas pour le Bundeskriminalamt (BKA) allemand et pour le Centrale Recherche Informatiedienst (CRI) néerlandais. Pour des pays comme l'Allemagne ou les Pays-Bas avec des structures de police relativement décentralisées, cela a déclenché la résistance des autorités régionales qui auraient préféré coopérer directement avec les centrales de Schengen et Europol. Finalement, les services centraux ont été renforcés et ils ont gagné de l'importance au détriment des structures décentralisées. Dans les pays avec plusieurs administrations policières concurrentes comme la France avec la Police Nationale et la Gendarmerie, la mainmise sur l'institution qui coopère avec Schengen et Europol était le sujet d'une rivalité dont le résultat a renforcé le poids des services centraux de l'une d'entre elles - de la Police Nationale dans le cas de la France ${ }^{14}$.

10 La coopération internationale des polices est elle-même une forme de centralisation parce qu'elle mène à des institutions comme Europol qui sont relativement éloignées de la vie quotidienne des citoyens et même des policiers " de base ». Au niveau national elle a favorisé une centralisation qui n'est pas identique avec une concentration de pouvoir. Des acteurs bureaucratiques du « top level » national ont gagné en importance par leur rôle de médiateurs entre les administrations décentralisées et les institutions de la coopération internationale. Ils ne peuvent développer le cadre général de leur travail que dans une coopération étroite avec les responsables politiques dont ils ont besoin pour assurer le cadre juridique et surtout financier de leurs activités. Si le processus de centralisation va encore plus loin avec des institutions européennes de sécurité qui reprennent une partie des missions réservées aux pays-membres jusqu'ici, cela pourra même affaiblir les acteurs policiers du «top level» national dans une perspective de longue durée.

Police et justice : élargissement du domaine autonome de la police

11 D'après le traité d'Amsterdam ${ }^{15}$ et les décisions du sommet de Tampere en octobre 1999, la coopération au niveau de la justice devrait avoir une priorité comparable à celle des polices. De fait, la coopération des polices est beaucoup plus avancée, ce qui est dû surtout aux initiatives des élites policières elles-mêmes. Si le traité d'Amsterdam prévoit l'élargissement des compétences d'Europol vers la coordination d'enquêtes communes $^{16}$, cela soulève la question de savoir si de telles fonctions ne nécessiteraient pas des éléments d'un droit pénal européen et surtout un parquet commun. En 1996 un premier pas avait été fait dans cette direction avec la mise en place d'un échange de magistrats de liaison suivant l'exemple des officiers de liaison dans le domaine de la police. Ce programme a été intégré dans une structure en réseau, le European Judicial Network. Après le sommet de Tampere, l'unité Eurojustcomposée d'un membre par pays 
a été mise en place, d'abord à Bruxelles avec un statut provisoire et depuis octobre 2002 à La Haye ${ }^{17}$. Elle réunit des procureurs, des juges d'instruction ou des fonctionnaires équivalents détachés par les États membres. Ces représentants ont pour mission de faciliter et de coordonner les investigations dans des cas concernant plusieurs pays et notamment dans ceux résumés sous le terme de crime organisé et basés sur les analyses d'Europol. Comme Europol, l'unité Eurojust est dotée de la personnalité juridique et dispose donc d'une autonomie considérable. Mais elle est encore loin des fonctions classiques d'un parquet. Le contrôle du travail d'Europol et la direction des enquêtes policières ne figurent pas encore sur la liste de ses missions.

Au niveau national, l'intensification de la coopération des polices a de fait renforcé la marge de manoeuvre autonome de la police criminelle par rapport à la justice. Dans le cas de l'Allemagne, comme dans bien d'autres pays, la procédure pénale et surtout les pratiques administratives laissent à la police judiciaire une autonomie considérable pendant l'instruction d'un cas. Les procureurs ne mènent des enquêtes eux-mêmes ${ }^{18}$ que pour les crimes graves et dans les cas difficiles. Dans tous les autres cas, la police judiciaire prépare le dossier d'une façon tout à fait autonome et ne l'envoie au parquet qu'après la fin de l'instruction. Même pour les crimes graves nécessitant une coopération renforcée entre police judiciaire et parquet ${ }^{19}$, c'est la police judiciaire qui fait la plus grande partie du travail. Les formes de coopération internationale qui impliquent les polices judiciaires et non la justice augmentent l'autonomie des institutions policières. Celles-ci se sont procurées par la coopération internationale de nouvelles sources d'informations et d'innovations techniques dont la justice ne dispose pas. Même si la justice voulait diriger les enquêtes policières, cela ne serait possible que de façon très limitée en raison de l'avance des policiers au niveau des ressources et du renseignement.

Les effets de synergie et de modernisation au niveau de la police technique

La coopération internationale des polices a déclenché des effets de synergie et de modernisation au niveau de la technique d'enquête. Les banques de données en fournissent un exemple évident. Le système informatique créé dans le cadre des accords de Schengen a connecté les banques de données préexistantes dans les pays membres. Les systèmes nationaux qui souvent n'étaient pas suffisamment performants pour fonctionner dans le cadre de ce réseau ont dû être adaptés ou même renouvelés. Le besoin de disposer d'un système informatique compatible avec le système Informatique Schengen (SIS)a accéléré la modernisation des systèmes informatiques des polices en vue de l'évolution technique. Dans la deuxième moitié des années 1990, les polices criminelles ont dû adapter leur matériel pour pouvoir se connecter à Europol. L'analyse de données sensibles par Europol n'est pas accessible pour les polices des pays membres. Mais les unités nationales ont le droit de consulter le système d'information général prévu dans l'article 7 de la convention Europol ${ }^{20}$.

La tendance va vers des systèmes qui fonctionnent en réseau. Ces systèmes intègrent les services de police nationaux et régionaux et assurent la connexion avec le SIS. Les polices allemandes au niveau fédéral et des Länder, par exemple, ont eu l'ambition ces dernières années de créer un tel système informatique intégré (INPOL-neu). En pratique, la mise en oeuvre de ce genre de réseau a mené à des problèmes importants. L'entrée en fonction des systèmes informatiques de Schengen et d'Europol fut reportée à plusieurs reprises. INPOL-neu, le projet allemand, n'a pas pu être réalisé dans la forme prévue initialement. L'idée d'assurer, en même temps, un haut niveau de sécurité et 
l'accès pour une multitude d'acteurs, d'après des critères différenciés, s'est révélée irréalisable.

Les impacts de la coopération internationale dans le domaine de la police technique ne se limitent pas aux banques de données. Pour d'autres techniques la tendance va également vers une harmonisation. Cela n'est pas seulement motivé par les avantages de la compatibilité des systèmes, mais aussi par l'intérêt financier à faire des économies en développant des systèmes utilisables dans plusieurs pays. Les systèmes d'identification automatique des empreintes digitales en sont un exemple. L'harmonisation de la technique policière est le résultat de la coopération dans une multitude de structures. En Europe, c'est notamment dans le cadre du groupe TREVI que cette harmonisation a été promue depuis les années $1970^{21}$. Depuis, cette coordination a continué dans le cadre des structures créées avec le troisième pilier de l'Union Européenne.

16 Au niveau national, cette harmonisation a augmenté le poids des services centraux. Ce sont eux qui assurent l'interconnexion entre les réseaux techniques internationaux et les services décentralisés dans leurs pays.

Les investigations coordonnées par Europol et l'extension des stratégies d'investigation « undercover»

17 Un autre impact de la coopération internationale est la coordination d'investigations par Europol. Déjà pratiquée au cas par cas depuis sa création, cette mission a été soulignée et renforcée par le traité d'Amsterdam de 1997 dont la mise en oeuvre est en cours.

Dès sa création, la coordination de livraisons surveillées a été une des missions de l'office Européen de Police Europol. Dans le cas d'une livraison surveillée, la police n'intervient pas le plus tôt possible quand elle reçoit des informations sur la préparation de la livraison d'un bien illégal, mais elle observe les faits pour connaitre d'autres éléments du réseau qui participent au crime. Plusieurs accords internationaux dont l'article 73 de l'accord complémentaire Schengen ont obligé les pays membres à rendre possible l'application de cette stratégie ${ }^{22}$. Pratiquées surtout pour les drogues illégales, les livraisons surveillées ont été étendues peu à peu vers d'autres domaines, par exemple vers les filières d'immigration clandestine ${ }^{23}$. La coordination d'investigations a de plus été renforcée depuis qu'il existe la possibilité d'installer au cas par cas une «task force » commune. L'accord sur l'assistance mutuelle conclu le 29 mai 2000 sur la base de l'article 34 du Traité sur l'Union Européenne ${ }^{24}$ et une décisioncadre du 13 juin $2002^{25}$ ont concrétisé cette stratégie.

19 Europol est encore loin des fonctions du Federal Bureau of Investigation (FBI) et des pratiques d'investigation « undercover ${ }^{26}$ aux États-Unis. La coopération des polices en Europe repose aujourd'hui sur un réseau de structures qui influence de plus en plus les pratiques des polices criminelles des pays membres, mais aussi celles des administrations qui gèrent l'immigration ${ }^{27}$. Par la voie d'accords internationaux, les pays qui y participent ont été forcés d'adopter de nouvelles formes d'investigation "undercover ». Au-delà de ces obligations formelles, l'utilisation de ce genre de stratégies d'investigation est devenue une norme internationale informelle. Les institutions de coopération ont contribué à disperser le savoir sur des stratégies comme les écoutes ou les infiltrations. L'impact sur les stratégies d'investigation au niveau national se résume donc avant tout par un gain en importance de l'investigation clandestine au détriment de l'investigation classique comme la recherche de témoins et 
d'autres stratégies de recherche de preuves. Même si la coopération elle-même ne change pas directement les procédures pénales des pays membres, l'évolution des stratégies d'enquête influence donc considérablement le travail quotidien de la police criminelle. Cela se reproduit pour les pays candidats à l'adhésion à l'Union Européenne ${ }^{28}$ qui se trouvent sous la pression d'adapter leurs stratégies d'investigation aux pratiques établies dans le cadre du troisième pilier.

Les effets au niveau du droit de la police : élargissement des bases légales

La coopération internationale a contribué à élargir les bases légales du travail policier au niveau national. L'exemple des livraisons surveillées de drogues et d'autres biens illégaux montre que l'adaptation des bases légales est dans bien des cas la conséquence directe d'un traité international. Plus généralement, c'est le cas pour l'ensemble de la politique de lutte contre ces drogues illégales pour laquelle les traités internationaux ont mené à une stratégie de sanctions pénales du trafic ou même de la possession des produits ${ }^{29}$.

21 Dans d'autres cas, l'extension des bases légales du travail policier au niveau national est une conséquence indirecte de la coopération internationale. C'est par exemple le cas pour les stratégies d'investigation « undercover » qui ont été légalisées successivement dans la plupart des pays européens. Pratiquées d'abord le plus souvent sans base légale explicite, les stratégies d'investigation clandestine se sont étendues à partir de l'exemple et parfois sous l'influence des polices des États-Unis ${ }^{30}$. En Europe, ces stratégies d'investigation ont dû être dotées de bases légales, surtout à cause de la jurisprudence de la Cour Européenne des Droits de l'Homme. La CEDH a constaté à plusieurs reprises que les investigations policières portant atteinte à la vie privée ${ }^{31}$ ne peuvent se justifier que sur la base d'une loi $^{32}$.

Un autre domaine dans lequel la coopération internationale a indirectement contribué à l'élargissement des bases légales du travail policier est celui des contrôles d'identité dans les régions frontalières. Les débats des années 1980 et 1990 sur des "mesures compensatoires " pour l'abolition des contrôles aux frontières internes entre les pays signataires des accords de Schengen ont contribué à l'extension des bases légales pour les contrôles d'identité, même sans suspicion concrète. La plupart des pays Schengen ont créé des bases légales pour ce genre de contrôles dans un corridor le long des frontières ou même dans des zones encore plus étendues, par exemple sur les autoroutes. Dans une autre logique de "compensation", les pays participant à Schengen et Europol ont dû créer une législationpour la protection des données qui auparavant n'avait d'existence que dans une partie des pays européens.

La conception du droit de la police est trop divergente dans les différents pays européens pour parler vraiment d'une tendance à l'uniformisation du droit dans ce domaine. Quelques polices travaillent sur la base d'une législation relativement détaillée qui définit les limites de leurs compétences par rapport aux citoyens. C'est par exemple le cas en Allemagne. Dans d'autres pays comme la France, le cadre juridique du travail policier reste assez général, surtout dans les domaines d'activité hors du champ de la procédure pénale ${ }^{33}$. La coopération des polices contribue à établir des normes internationales qui mènent à une harmonisation des bases légales du travail policier dans les secteurs concernés par la coopération.

Les effets sur la criminalité : définition de nouveaux problèmes et difficultés de mesurer les effets sur l'élucidation des crimes constatés 
24 Les effets que la coopération internationale peut avoir sur la criminalité sont aussi difficiles à mesurer que les impacts du travail policier en général ${ }^{34}$. La menace que « le crime international » ou le « crime organisé international » constituent pour la sécurité est l'élément central du discours justifiant la nécessité d'un renforcement de la coopération internationale. En fonction de la "conjoncture " s'y ajoutent d'autres figures de légitimation comme le terrorisme ou l'immigration illégale. Dans le discours des élites policières et des responsables politiques, ces menaces restent relativement abstraites. Même si certains exemples, comme celui du trafic de drogues illégales sont souvent cités, le crime organisé est plutôt resté une construction dont le contenu concret n'est pas clairement défini ${ }^{35}$. De plus, les pratiques de la déviance varient et évoluent ${ }^{36}$. Petits et grands criminels se servent des inventions techniques et changent leurs pratiques.

25 L'ambition de quantifier les effets que la coopération internationale des polices peut avoir sur la criminalité se heurte donc surtout à ce problème : une bonne partie des phénomènes traités dans ce cadre n'est venue à l'ordre du jour que par cette coopération elle-même. Quelques-uns des phénomènes de criminalité traités n'étaient auparavant pas connus ou reconnus en tant que tels dans les différents pays. Dans ces cas, il est impossible de mesurer les effets de la coopération internationale, car on ne connaît pas l'étendue exacte que ces problèmes peuvent avoir eu auparavant. Parfois, les problèmes traités par les institutions de coopération peuvent même se révéler être une pure construction. C'est par exemple le cas pour le trafic illégal de plutonium qui s'était trouvé subitement à l'ordre du jour des institutions de la coopération policière au milieu des années 1990. Peu de temps après, il est apparu qu'une bonne partie de ce trafic avait été mise en scène par des services secrets ${ }^{37}$. Dans ce genre de cas, bien entendu, les activités développées pour les «combattre » ne peuvent pas avoir d'effet réel sur la sécurité.

En outre, les activités policières contre le «crime organisé international » peuvent avoir des effets statistiques ambigus. Si la police saisit une certaine quantité de drogues illégales et arrête les dirigeants d'une filière de trafic, cela ne veut pas automatiquement dire que la consommation de drogues baisse. Il se peut aussi bien que l'arrestation d'un acteur du marché noir ne mène qu'à une hausse des prix au profit des autres marchands du même bien illégal. Cet effet peut se produire pour tout genre de trafic illicite.

Les impacts de la coopération internationale sur les professions policières

Même si la coopération internationale des polices n'implique actuellement directement qu'une petite partie des policiers, elle contribue à changer les professions policières dans une perspective de longue durée. Le prototype du policier international est l'officier de liaison qui représente "sa " police auprès d'un autre pays ou d'une institution de la coopération internationale ${ }^{38}$. Bien évidemment, il ne s'agit que d'une petite élite.

Au-delà de cette élite la maîtrise de langues étrangères est désormais indispensable pour tous les cadres supérieurs. La connaissance de langues est devenue un critère de recrutement. La formation des hauts et moyens fonctionnaires de police inclut des éléments d'ouverture internationale. Cette tendance a été renforcée par une coopération officielle dans le cadre de la formation initiale et permanente des policiers. Le groupe TREVI proposait déjà des séminaires communs pour les cadres supérieurs. Ces activités ont été poursuivies dans le cadre du troisième pilier de l'Union 
européenne. En décembre 2000, le Conseil de l'Union Européenne décida de la création d'un Collège européen de Police (Cepol) ${ }^{39}$. Dans un premier temps, il s'agit d'une structure de coordination et de coopération des instituts nationaux de formation des hauts responsables de police, ouverte également aux pays candidats à l'adhésion à l'Union Européenne. L'objectif est de coordonner la formation des hauts fonctionnaires de police et les programmes de formation continue en vue de la coopération internationale. Dans le domaine de la formation s'est donc imposée, au moins provisoirement, une structure en réseau au lieu d'une structure centralisée comme elle a été instituée dans le domaine de la police criminelle avec Europol.

Certains types de coopération en dehors de la police criminelle contribuent également au rapprochement des professions policières dans les différents pays européens. C'est par exemple le cas pour la coopération au quotidien dans les régions frontalières. La coopération des unités spécialisées dans l'accompagnement d'événements majeurs comme les championnats internationaux de football ou l'exposition universelle qui a eu lieu en Allemagne en 2000, intègre également de plus en plus des policiers de base. Des programmes d'échange ont contribué à élargir le cercle des policiers impliqués dans ces formes de coopération.

L'orientation internationale du travail policier favorise le type pragmatique du policier qui est le « crime fighter » au sein de la police criminelle. Les valeurs traditionnelles du travail policier comme l'habilité sportive, l'habilité au tir et les connaissances du droit pénal perdent de l'importance. La maîtrise des outils électroniques de communication et d'enquête est essentielle pour le nouveau type de policier qu'on trouve de plus en plus dans tous les pays européens.

Nouvelles difficultés pour un contrôle démocratique du travail policier au niveau national et international

31 La coopération internationale affecte la possibilité de contrôler les activités des polices. Si une institution devient plus complexe son contrôle devient plus difficile. Cela est le cas pour la coopération internationale qui augmente la complexité des structures de police. Au niveau national, régional et même au niveau local déjà, le travail policier est difficile à contrôler par les différents acteurs qui pourraient s'y apprêter. Le contrôle par les parlements et même celui exercé par la justice se heurtent traditionnellement au secret professionnel qui caractérise certains aspects du travail policier, notamment l'investigation pénale.

La coopération internationale est une forme d'interconnexion à plusieurs niveaux. Cette interconnexion limite la possibilité des institutions implantées à un seul niveau administratif d'exercer un contrôle efficace. La justice et les parlements nationaux se heurtent à la complexité de la coopération à contrôler qui dépasse en principe leur champ géographique de compétence. Vice versa, le Parlement Européen et les parlements nationaux ne peuvent contrôler les activités des institutions de coopération que d'une façon très limitée - non pas seulement parce qu'Europol et les autres institutions de coopération gardent secrète une bonne partie de leurs activités, mais aussi parce que les interconnexions avec le travail policier national et régional rendent le contrôle très difficile. Le contrôle par un public spécialisé se heurte aux mêmes difficultés ${ }^{40}$.

33 Pour répondre à ce genre de difficultés, Schengen et Europol ont créé des mécanismes de contrôle qui fonctionnent eux-mêmes dans une logique de multi-level governance. Les groupes d'experts qui assurent le contrôle de la protection des données par Schengen 
et Europol sont composés de délégués des institutions nationales de protection des données. Mais ce contrôle reste limité au domaine de la protection des données. La disposition de la Convention Europol qui donne au Parlement Européen le droit à un rapport annuel sur les activités d'Europol (art. 34) semble insuffisante pour assurer un contrôle réel de la part des députés européens. Ce n'est donc pas par hasard si les possibilités de renforcer le contrôle des activités d'Europol et de coordonner les approches de contrôle au niveau national et européen font toujours l'objet d'un débat vif $^{41}$.

En conclusion, l'on pourra retenir que la coopération internationale des polices ne change pas d'une façon soudaine ou même révolutionnaire le travail policier au niveau national, régional et local. Mais elle a un certain nombre d'impacts qui modifient peu à peu le « policing".

Sous certains aspects le travail policier a tendance à évoluer vers des structures de multi-level governance dans lesquelles la répartition des rôles reste à définir : dans la dimension verticale entre les niveaux politiques et administratifs européens, nationaux et régionaux et dans la dimension horizontale entre les élites policières, la justice, le pouvoir politique et des fois des acteurs privés. On peut prévoir que dans le domaine de la sécurité, la coordination et la coopération vont continuer à gagner en importance avec l'ouverture de l'Union Européenne aux pays d'Europe centrale et les prochains pas de l'intégration européenne qui pourraient suivre. La coopération dans le domaine de la sécurité publique ne fonctionne pas d'après les mêmes logiques que l'intégration européenne dans ses domaines classiques du premier pilier $^{42}$. Il faudra faire un choix entre des formes d'intégration renforcée et la continuation d'une coopération plutôt intergouvernementale comme elle a été pratiquée au sein du troisième pilier de l'UE depuis l'accord de Maastricht. Une structure de coordination en réseau telle qu'elle a été choisie dans le cas du Collège européen de Police a l'avantage de mieux pouvoir intégrer l'expérience des institutions établies au niveau national et d'éviter les pièges d'une méga-bureaucratie coûteuse et éloignée des besoins du travail policier au quotidien. Mais l'intérêt des responsables politiques à disposer d'institutions auxquelles ils peuvent déléguer la responsabilité de la gestion de nouveaux risques d'insécurité semble plutôt favoriser les bureaucraties centralisées comme Europol.

Un système de police basé sur l'interconnexion entre différents niveaux politiques et administratifs nécessite des structures assurant à la fois un travail policier efficace et un esprit démocratique au sein duquel les institutions de sécurité doivent nécessairement s'orienter vers un maximum de transparence de leurs activités et dans lequel un système de contrôle ne soit pas perçu comme de la méfiance envers les policiers mais comme un élément indispensable d'un système de police moderne et performant. 


\section{NOTES}

1. . Je remercie Sylvie Zemb (CESDIP/CNRS) pour la révision linguistique de ce texte. J'en ai présenté une version antérieure lors du séminaire « Questions de Police/Policing Matters » à Paris, 23-24 mars 2001, organisé par Jean-Marc Berlière, Clive Emsley, René Lévy et Dominique Monjardet dans le cadre du Groupe Européen de Recherches sur les Normativités (GERN/CNRS). Je remercie les participants de ce séminaire et les lecteurs anonymes de Cultures \& Conflits de leurs commentaires.

2. . Voir par exemple Marks G., Hooghe L., Blank, K., « European Integration from the 1980s : State-Centric v. Multi-level Governance » in Journal of Common Market Studies 1996 (Vol. 34, No. 3), pp. 341-378.

3. . Art. 10 Convention Europol du 26 juillet 1995, JO CE C 316, pp. 25ss.

4. . Art. 9 Convention Europol sur la mise en oeuvre : Manske M., Das, « EuropolInformations-System » (Europol-IS), in Kriminalistik 2001, pp. 105-108.

5. . Dans le sens d'un système « autopoétique » d'après Luhmann N., Das Recht der Gesellschaft, Frankfurt/Main, Suhrkamp, 1993, pp.42ss.

6. . Voir Brammertz S., De Vreese S., Thys J., Internationale Politiesamenwerking. Onderzoek naar de modaliteiten voor Belgische participatie in internationale poltiesamenwerkingsprojecten van regionale omvang, Bruxelles, Politeia, 1993, p. 29 ; Aden H., Polizeipolitik in Europa. Eine interdisziplinäre Studie über die Polizeiarbeit in Europa am Beispiel Deutschlands Frankreichs und der Niederlande, Opladen/ Wiesbaden, Westdeutscher Verlag, 1998, pp. 74-76.

7. . Voir Aden H., Polizeipolitik in Europa, op.cit., pp. 229ss.

8. . Ibid., pp. 46 et passim.

9. . Benz A., Föderalismus als dynamisches System : Zentralisierung und Dezentralisierung im föderativen Staat, Opladen (Westdeutscher Verlag) 1985, pp. 120 et passim.

10. . Fijnaut C., Opdat de macht een toevlucht zij ? Een historsiche studie van het politieapparaat als een politieke instelling, Arnhem et Antwerpen (Gouda Quint et Kluwer Rechtswetenschappen) 1979, pp. 436-438.

11. . Dans une perspective comparative : Aden H., Polizeipolitik in Europa, op.cit., pp. 119 et passim.

12. . Supplementary Information Request at the National Entry ; art. 92 II Accord Complémentaire Schengen du 19 juin 1990 pour le SIS.

13. . Art. 4 Convention Europol.

14. . Aden H., Polizeipolitik in Europa, op.cit., pp. 48-64 et 101-105.

15. . Articles 29 et 31 (ex-art.K.1 et K.3) du traité sur l'Union Européenne (UE).

16. . Article 30 UE.

17. . D'abord avec la Décision du Conseil de l'UE du 14 décembre 2000, JO CE L 324 du 21/12/2000, pp. 2s. sous le titre de Pro-Eurojust, remplacée par la Décision du Conseil de l'UE du 28 février 2002, JO CE L 63 du 6/3/2002, pp. 1ss. ; la coopération dans le cadre d'Eurojust est également prévue dans les articles 29 et 31 du traité sur l'Union Européenne dans la rédaction nouvelle du traité de Nice.

18. . Voir $n^{\circ} 3$ des Richtlinien für das Strafverfahren und das Bußgeldverfahren (RiStBV) - règlement administratif des ministres de la justice. 
19. . Voir Pütter N., Der OK-Komplex. Organisierte Kriminalität und ihre Folgen für die Polizei in Deutschland, Münster (Westfälisches Dampfboot) 1998, pp. 261ss.

20. . Sur la genèse : Masson $P$., Europol et la lutte contre les trafics de drogues, Paris (Sénat français, rapport $n^{\circ}$ 235/1994-1995) 1995, pp. 19ss.

21. . Aden H., Polizeipolitik in Europa, op.cit., pp. 76ss.

22. . Sur la pratique et les définitions juridiques de cette stratégie : Bevers H., Joubert

Ch., Schengen investigated. A Comparative Interpretation of the Schengen Provisions on International Police Cooperation in the Light of the European Convention on Human Rights, Deventer, Kluwer, 1996, pp. 189ss. ; Busch H., Polizeiliche Drogenbekämpfung eine internationale Verstrickung, Münster, Westfälisches Dampfboot, 1999, pp. 266ss.

23. . Europol, Annual report 2000, La Haye 2001, pp. 4 et 20.

24. . JO CE 2000, C 197.

25. . JO CE 2002, L 162.

26. . Cf. sur les dimensions de ces pratiques aux Etats-Unis : Marx G. T., Undercover : Police surveillance in America, Berkeley, Los Angeles, University of California Press, 1988.

27. . Sur ce réseau : Hayes B., The activities and development of Europol - towards an unaccountable «FBI » in Europe, Londres, Statewatch publications, 2002, p. 15 et passim.

28. . Sur les préalables de l'adhésion : Bigo D., « La coopération policière avec les PECO entre confiance et exigence ", Les Cahiers de la Sécurité Intérieure no. 41 (mars 2000), pp. 141ss.

29. . In extenso : Busch, op.cit., pp. 52ss.

30. . Cf. Nadelmann E.A., Cops across Borders. The Internationalization of US Criminal Law Enforcement, Pennsyslvania, The Pennsylvania State University Press, 1993.

31. Protégée par l'article 8 de la Convention européenne des Droits de l'Homme.

32. . Sur cette jurisprudence : Bevers H., Joubert Ch., op.cit., pp. 518s.

33. . Sur les causes : Aden H., « Der Etat de droit der V. französischen Republik und seine Begrenzung durch große exekutive Handlungsspielräume » in Deutsch-

Französisches Institut Albertin L., Asholt W. et al. (éd.), Frankreich-Jahrbuch 2001, Opladen (Leske + Budrich) 2001, pp. 65ss.

34. . Sur ce vieux problème voir par exemple : Skolnick J. H., Justice without Trial, New York/Londres, Wiley \& Sons, 1966, pp. 164ss. ; Kerner H.-J., « Kriminalstatistik », in Kaiser G., Kerner H.-J., Sack F., Schellhoss H., Kleines Kriminologisches Wörterbuch, 3ème tirage, Heidelberg (C.F. Müller) 1993, pp. 294-301.

35. . Cf. Aden H., « Europäische Polizeikooperation - Konstruktion und Wandel von Legitimationsfiguren » in : Hitzler, R./Peters, H., Inszenierung : Innere Sicherheit. Daten und Diskurse, Opladen, Leske + Budrich, 1998, pp. 65ss.

36. . Cf. Bigo D., Polices en réseaux, Paris, Presses de Sciences Po, 1996, pp. 254ss. 37. . Exemples pour ce genre de menace irréelle : Aden H., Polizeipolitik in Europa, op.cit., pp. 143s.

38. . Voir Bigo D., « Liaison officers in Europe : new officers in the European security field » in Sheptycki J. (éd.), Issues in Transnational Policing, London, New York, Routledge, 2000, pp. 67ss.

39. . Décision du Conseil de l'UE du 22 décembre 2000, JO CE L 336 du 30 décembre 2000, pp. 1-3.

40. . Voir Aden H., Polizeipolitik in Europa, op.cit., pp. 349ss. 
41. . Voir par ex. Commission Européenne, Le contrôle démocratique d'Europol, Bruxelles (KOM(2002)95), 26 février 2002.

42. . Cf. Knelangen W., Das Politikfeld innere Sicherheit im Integrationsprozess, Opladen, Leske + Budrich, 2001.

\section{RÉSUMÉS}

La coopération internationale des polices a mené à deux types d'institutionnalisation : D'un côté, à Europol, Interpol et d'autres cercles de coopération de la police criminelle qui sont relativement éloignés du travail policier au quotidien. De l'autre côté à de multiples formes de coopération dans les régions frontalières. Ces deux types de coopération sont aujourd'hui des éléments d'une forme spécifique de multi-level governance politique et administratif dans le domaine de la sécurité publique : Les structures de coopération sont influencées par les polices et les politiques préexistantes au niveau national et régional. Dans le sens inverse, la coopération interna-tionale a un certain nombre d'impacts au " policing » national, régional et même local, notamment pour la police criminelle. Elle a par exemple eu des effets centralisateurs, elle a augmenté l'autonomie policière par rapport à la justice et elle favorisé le gain d'importance des formes d'investigation « undercover».

International police cooperation has brought up two basic forms of institutionalisation: On the one hand Europol, Interpol and other cooperation circles of the criminal police which are relatively far away from daily policing in the member states. And on the other hand various forms of cooperation in the border re-gions. These two basic forms of cooperation are elements of a specific administrative and political form of multi-level gov-ernance: International cooperation is being influenced by na-tional and regional administrations and politics. And, the other way round, international cooperation has a number of impacts on national, regional and even local policing. Cooperation has for example contributed to the centralisation of policing, it has enlarged police autonomy in relation to criminal justice and it has contributed to the dissemination of undercover policing.

\section{INDEX}

Index géographique : Europe

Mots-clés : coopération internationale, police

Thèmes : EUROPOL 